\title{
Malaria control along China-Myanmar Border during 2007-2013: an integrated impact evaluation
}

Jian-Wei Xu ${ }^{1 *}$, Yong Li ${ }^{2}$, Heng-Lin Yang ${ }^{1}$, Jun Zhang ${ }^{3}$, Zai-Xing Zhang ${ }^{4}$, Ya-Ming Yang ${ }^{1}$, Hong-Ning Zhou ${ }^{1}$, Joshua Havumaki ${ }^{5}$, Hua-Xian Li ${ }^{1}$, Hui Liu ${ }^{1}$, Hua Zhou ${ }^{2}$, Xin-Yu Xie ${ }^{3}$, Jia-Xiang Dong ${ }^{3}$, Yue Zhang ${ }^{3}$, Xiao-Ying Sun ${ }^{3}$, Bo Li $i^{3}$, Jia-Yin Li ${ }^{3}$, Yang-Hui Tian ${ }^{3}$, Pi-Yu Wang ${ }^{1}$ and Ben-Fu Li ${ }^{1}$

\begin{abstract}
Background: Implementing effective interventions remain a lot of difficulties along all border regions. The emergence of artemisinin resistance of Plasmodium falciparum strains in the Greater Mekong Subregion is a matter of great concern. China has effectively controlled cross-border transmission of malaria and artemisinin resistance of $P$. falciparum along the China-Myanmar border.

Methods: A combined quantitative and qualitative study was used to collect data, and then an integrated impact evaluation was conducted to malaria control along the China-Myanmar border during 2007-2013.

Results: The parasite prevalence rate (PPR) in the five special regions of Myanmar was decreased from $13.6 \%$ in March 2008 to $1.5 \%$ in November 2013. Compared with the baseline (PPR in March 2008), the risk ratio was only 0.11 [95\% confidence interval $(C D), 0.09-0.14)$ in November 2013, which is equal to an $89 \%$ reduction in the malaria burden. Annual parasite incidence (API) across 19 Chinese border counties was reduced from 19.6 per 10000 person-years in 2006 to 0.9 per 10000 person-years in 2013. Compared with the baseline (API in 2006), the API rate ratio was only 0.05 (95\% Cl, 0.040.05) in 2013, which equates to a reduction of the malaria burden by $95.0 \%$. Meanwhile, the health service system was strengthened and health inequity of marginalized populations reduced along the international border.
\end{abstract}

Conclusion: The effective collaboration between China, Myanmar and the international non-governmental organization promptly carried out the core interventions through simplified processes. The integrated approaches dramatically decreased malaria burden of Chinese-Myanmar border.

Keywords: Border malaria, Control, Artemisinin resistance, China-Myanmar border

\section{Multiligual abstracts}

Please see Additional file 1 for translations of the abstract into the six official working languanges of the United Nations.

\section{Background}

A population of 3.2 billion is currently at risk for malaria infection on the globe [1]. The Greater Mekong

\footnotetext{
* Correspondence: xjw426@163.com

${ }^{1}$ Yunnan Institute of Parasitic Diseases, Yunnan Provincial Center of Malaria Research, Yunnan Provincial Collaborative Innovation Center for Public Health and Disease Prevention and Control, Yunnan Provincial Key Laboratory of Vector-borne Diseases Control and Research, Puer 665000, China

Full list of author information is available at the end of the article
}

Subregion (GMS) is considered a hot-spot of malaria transmission with around $70 \%$ of the local population at risk of contracting malaria $[2,3]$. Within the GMS, China has made extraordinary progress in malaria control [4] and aims to eliminate malaria nationwide by 2020 [5]. On the other hand, Myanmar, which shares a border of $2185 \mathrm{~km}$ with China, is still among the 31 highest burden malaria countries in the world [2, 6]. Importantly, Myanmar exports malaria to its neighboring countries. This seriously impedes progress of malaria elimination in these countries [7]. Additionally, five of the six GMS countries, all except China, have reported artemisinin resistance of Plasmodium falciparum $[1,8-12]$. 
Malaria control and elimination together with attempts to contain drug resistance have received much attention along international borders, but effective interventions remain challenging $[1,5,8]$. From 2007 to 2013, intensive efforts have dramatically reduced the number of malaria cases and the parasite prevalence along China- Myanmar border; meanwhile, the drug sensitivity of malaria parasites has not changed significantly $[13,14]$. Knowledge and lessons learnt from the border malaria control would benefit the global health. In this context, an impact evaluation was conducted on the cross-border malaria control program.

\section{Methods}

\section{Study site and population}

This study was conducted between January 2008 and May 2014 across 19 border counties of Yunnan Province in China and five special regions in Myanmar (Fig. 1). The study population consisted of 4687896 Chinese individuals and 586000 residents from Myanmar. Notably, there were estimated 110000 longer term migrants $(\geq$ one month in Myanmar) from China based on the five Special Regions of Myanmar, and 1.5 million short-term migrants from both China and Myanmar who cross the border frequently. More than $50 \%$ of the study population was ethnic minorities [15]. These groups could not usually receive sufficient access to healthcare. Malaria control services of the Myanmar Ministry of Health were unable to effectively cover the five special regions due to ongoing conflicts between the Governments of Union of Myanmar and locally ethnical military.

\section{Core interventions}

The sixth and tenth rounds of the Chinese malaria programs within the Global Fund to fight AIDS, Tuberculosis and Malaria (GFATM) launched in July 2007. The GFATM program established 68 border malaria posts (BMP) at informal border crossing locations throughout the 19 Chinese counties. The BMPs provided diagnosis and treatment for malaria, long lasting insecticidal nets (LLIN) and behavior change communication (BCC) for the 1.5 million short-term migrants. The existing public health facilities were responsible for malaria control of the local residents across 19 border counties in China.

Health Poverty Action (HPA), an UK-based nongovernment organization (NGO), established 80 malaria diagnoses and treatment stations (MDTS) in the five special regions of Myanmar. The MDTs provided diagnosis and treatment for the residents of Myanmar and the Chinese longer term migrants who were based in the five special regions of Myanmar. In order to address gaps in public sector coverage in Myanmar, the GFATM programs supported community-based services, including, diagnosis and treatment in 102 selected private clinics in communities where MDTS were not available, 475 village malaria workers (VMW) in remote and hyperendemic villages and 30 migrant malaria workers (MMW) in the communities of Chinese longer term migrants. Meanwhile, HPA ran outreach services incorporating LLIN distribution, BCC and support supervision together. The outreach teams trained peer health educators in villages, and supervised operations of MDTS, VMWs and MMWs and gave technical support to them. HPA also worked with the local governments to introduce regulations banning the sale of the fake and sub-standard antimalarial drugs including artemisinin monotherapy.

\section{Study procedures}

Data of process evaluation were collected monthly. In China, the malaria case and its related information such as demographic data and travel history (for identifying the place of infection), were daily collected and reported by "Chinese Information System for Disease Control and Prevention (CISDCP)" [16]. The travel history was used to identify the infection origin in those cross border people. Malaria patients that had once been in Myanmar within one month prior to laboratory diagnosis were defined as imported malaria [5].

In five special regions of Myanmar, HPA's Health Information System which was established and maintained by the GFATM programs collected and reported the number of malaria cases every month. Meanwhile, cross-sectional surveys were conducted to collect data on parasite prevalence among residents between January and March in 2008, 2009, 2010 and 2011; and between September and November in 2012 and 2013. The study design was fixed as described by Wang et al. [17].

The GFATM program supplied antimalarial drugs. Dihydroartemisinin-piperaquine (DP) is the most commonly used artemisinin- based combination therapy (ACT) for treatment of $P$. falciparum malaria. Chloroquine was commonly used for treatment of $P$. vivax malaria. The sensitivity of $P$. falciparum to DP and the sensitivity of $P$. vivax to chloroquine were in vivo monitored [13, 14, 18-20].

From November 2013 to May 2014, qualitative study consisting of focus group discussions (FGD) and semistructured in-depth interviews (SDI) were undertaken to collect qualitative data for the beneficiary- and community-based evaluation [21-23]. Four FGDs were planned for stakeholders and eight FGDs for villagers, 8-10 participants each group. SDIs were planned to administer to 26 key informants. The issues discussed with participants in FGDs and key informants of SDIs included change of malaria burden; impact on public health, healthcare system and social equity; and finally, challenges and recommendations for malaria control in the border region. 


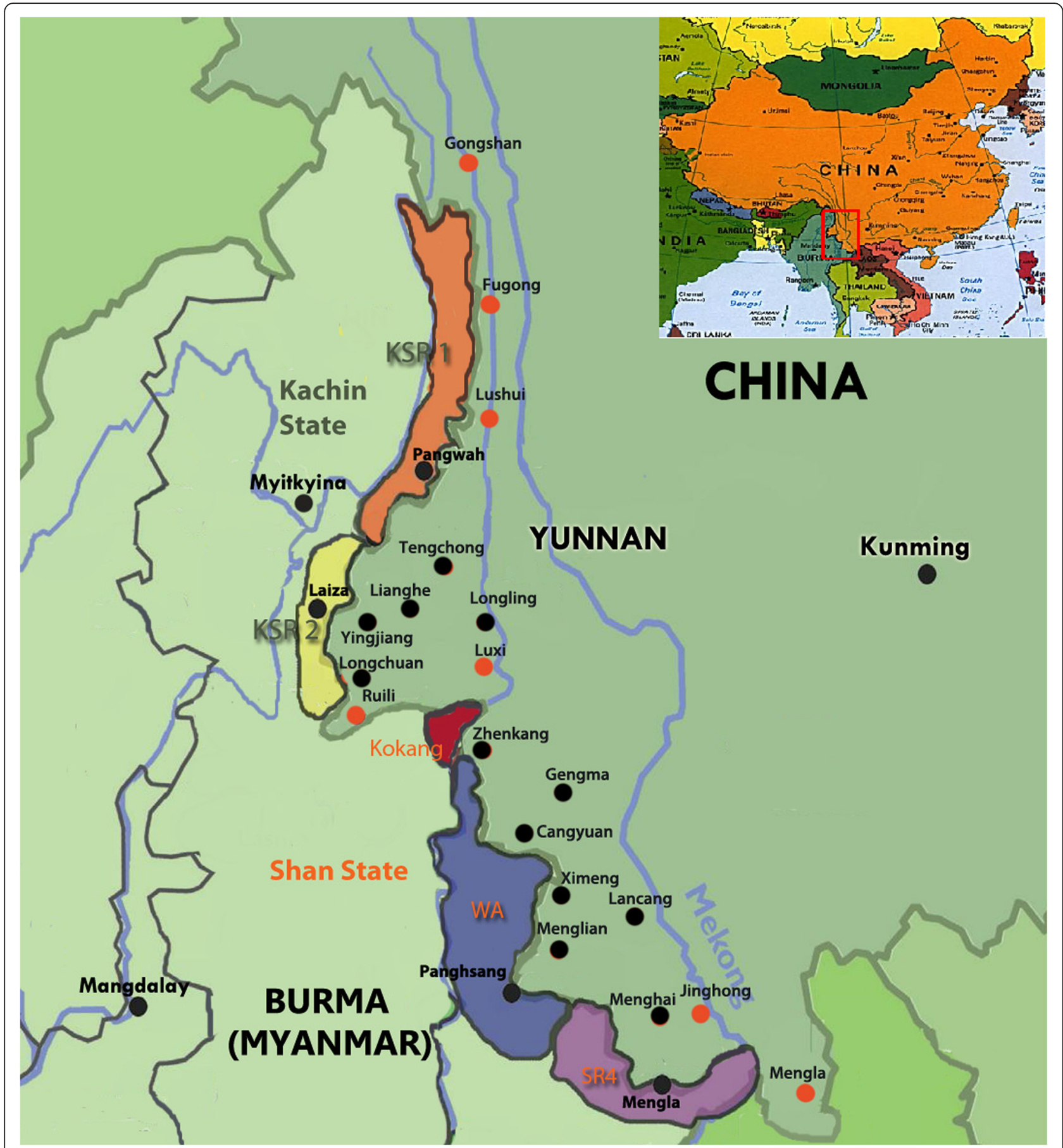

Fig. 1 Map of the study site and neighboring region

\section{Statistical analysis}

The data of core interventions were aggregated and analyzed every six months. The parasite prevalence rate (PPR) and the annual parasite incidence (API) with $95 \%$ confidence intervals $(C I)$ were calculated by Epi Info 7 (Centers for Disease Control and Prevention, Atlanta, GA). Risk ratios (RR) with $95 \% C I$ in Myanmar were calculated by using the PPR in March, 2008 as the reference. API rate ratios (IRR) with $95 \% C I$ were calculated by taking the API of 2008 in Myanmar and the API of 2006 in China as the reference respectively. Qualitative data analysis was conducted by software TAMS 3.0. The data were encoded based on emerging themes and then a codebook was created. Trends in the data were identified by producing matrices which combined and compared with information collected from the different FGDs and key informants. 
Finally, quantitative and qualitative results were combined and compared [21-23].

\section{Results}

\section{Core interventions}

Core interventions were successfully targeted residents along the China-Myanmar border. Table 1 indicates the numbers of core interventions, including LLINs distributed, diagnostic tests used, short-term migrants who received piperaquine for preventive treatment, cases treated with quality-assured ACTs and chloroquine- primaquine (CQ-PQ).

\section{Reduced malaria burden in Myanmar}

The malaria burden was gradually reduced since the start of the GFATM interventions in 2008. In Myanmar, the PPR decreased from $13.6 \%$ (95 \% CI, 12.7-14.6\%) in March, 2008 to 1.5 \% (95 \% CI, 1.2-2.0 \%) in November, 2013 (Fig. 2). Compared with the baseline (PPR in March 2008), the RR was only $0.11(95 \% C I, 0.09-0.14)$ in November 2013, which is equal to an $89 \%$ reduction in the malaria burden (Table 2). Additionally, the API reduced from 417.4 (95\% CI, 408.6-426.3) per 10, 000 person-years in 2008 to 71.2 (95\% CI, 69.2-73.4) per 10, 000 person-years in 2013 (Fig. 3). Compared with the API in 2008, API rate ratio (IRR) was only 0.17 (95\% CI, 0.16-0. 18) in 2013 (Table 3). There was not any reported malaria-related death during 2008-2013.
In Kachin Special Region II (KR2), stakeholders of FGDs still listed malaria as the most important public health problem. However, they said that the number of malaria cases had been reduced dramatically, and that there were no malaria-related deaths after intervention. Participants of internally displaced people in Je Yang Hka and Hpun Lum Yang said that "Malaria was the most life-threatening disease before intervention in KR2. For instance, there were more than 20 deaths of malaria in Ban Dong, a village with approximately 4000 residents in 2006".

In eastern Shan Special Region IV (SR4), stakeholders of FGDs described malaria as the most important public health problem before the intervention. However, malaria was just listed as the fourth or fifth one following the intervention. Participants considered malaria as the most burdensome communicable disease. They said that $P$. falciparum malaria prevalence and mortality were very high before the intervention, and currently, morbidity was very low. The deputy director of the health department of SR4 said that "the proportion of malaria was about 40$60 \%$ of outpatients before 2006". The chief of the Health Bureau of Nan Bang County of SR4 said that "many people died of malaria prior to the intervention, and then various people left Nan Bang because of malaria fearfulness. However, malaria is uncommon now; there is only one or two imported malaria cases from other areas of Myanmar". Participants of Man Beng Long and Man Beng Mai villages said that: "Malaria was the most common

Table 1 Amount of core interventions across Chinese-Myanmar border from 2008 to 2013

\begin{tabular}{|c|c|c|c|c|c|c|c|c|}
\hline Core interventions & 2007 & 2008 & 2009 & 2010 & 2011 & 2012 & 2013 & Total \\
\hline \multicolumn{9}{|c|}{$\begin{array}{l}\text { Five special regions of Myanmar (Target populations: a population of } \\
586000 \text { local residents and about } 110000 \text { Chinese immigrants in Myanmar) }\end{array}$} \\
\hline Numbers of long-lasting insecticidal bed nets distributed & 13866 & 67753 & 25274 & 13943 & 38134 & 84506 & 93501 & 336977 \\
\hline $\begin{array}{l}\text { Number of febrile patients who received parasite-based diagnosis } \\
\text { (RDTs or microscopy) }\end{array}$ & 10706 & 116163 & 130684 & 103538 & 26431 & 71289 & 121083 & 579894 \\
\hline Number of malaria cases treated with ACTs & 3059 & 27254 & 37192 & 25782 & 2084 & 8510 & 11537 & 106908 \\
\hline Number of malaria cases treated with CQ-PQ & - & 193 & 18339 & 24580 & 5112 & 3731 & 6642 & 53485 \\
\hline \multicolumn{9}{|l|}{ Health education } \\
\hline Number of community health education sessions organized & - & 537 & 579 & 637 & 301 & 187 & 651 & 2892 \\
\hline Number of attendees of community health education sessions & - & 47560 & 40099 & 42801 & 19218 & 10657 & 28892 & 189227 \\
\hline \multicolumn{9}{|l|}{$\begin{array}{l}19 \text { counties of China (Target populations: border-crossing migrants, } \\
\text { about 1,500,000 person-times each year) }\end{array}$} \\
\hline Numbers of long-lasting insecticidal nets distributed to migrants & 9486 & 30867 & 10977 & 16275 & 20042 & 66922 & 31031 & 185600 \\
\hline Number of migrants who received preventive treatment & 28976 & 37552 & 47594 & 44479 & 27719 & 46740 & 44269 & 277329 \\
\hline $\begin{array}{l}\text { Number of migrant febrile patients who received Parasite-based } \\
\text { diagnosis (Microscopy) }\end{array}$ & 41126 & 75495 & 106234 & 91332 & 70040 & 70690 & 67168 & 522085 \\
\hline Number of migrant malaria cases treated with ACTs & 808 & 853 & 1644 & 1184 & 546 & 207 & 125 & 5367 \\
\hline Number of migrant malaria cases treated with CQ-PQ & 3056 & 7081 & 8699 & 6044 & 2083 & 1084 & 1220 & 29267 \\
\hline Number of migrants who received IEC materials & 36649 & 152989 & 88453 & 98295 & 161194 & 108542 & 211316 & 857438 \\
\hline
\end{tabular}




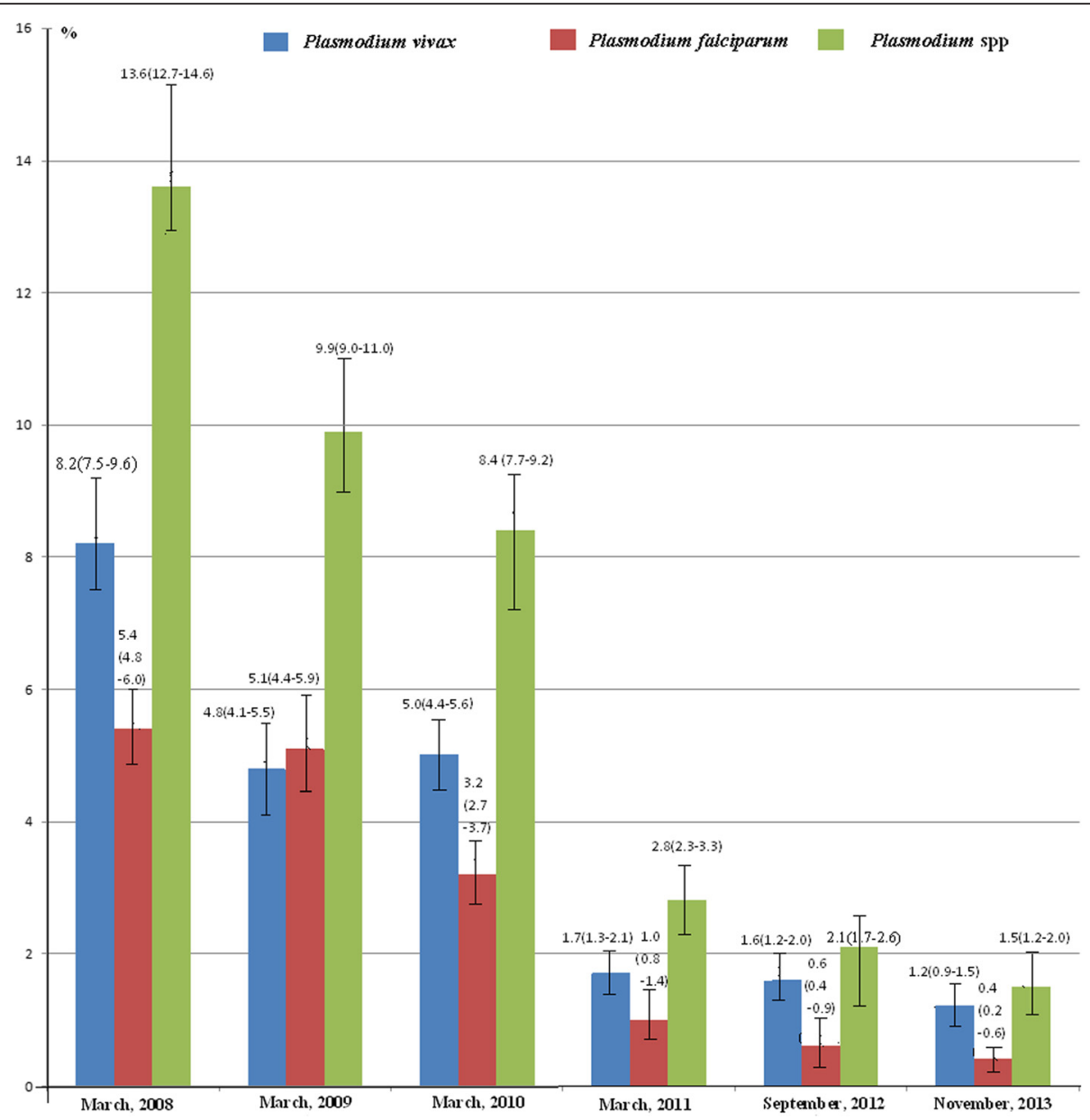

Fig. 2 Parasite prevalence rates in the five special regions of Myanmar from March 2008 to November 2013

disease prior to the intervention, $40 \%-50 \%$ of people contracting malaria each year. Some families had three or four malaria patients a year. However, malaria was currently scarce. It was difficult to find a malaria patient among 100 people. Only men who ever worked in the forest, such as lumbering workers, could contract malaria".

In Shan Special Region II (locally called Wa State), the director of the health department said that "many intervention activities had been carried out in Wa State. The malaria prevalence was very low after these interventions". The project manager of HPA of Wa State said that: "About twenty percent of febrile patients were malaria prior to the intervention. In 2005, more than 300 malaria cases were confirmed by Rapid diagnosis test (RDT) during three work days in Lian He Township. However, it was difficult to find malaria cases at all there following the intervention".

Table 2 Change of risk ratios in five special regions of Myanmar from 2008 to 2013

\begin{tabular}{|c|c|c|c|c|c|c|c|c|c|}
\hline \multirow[t]{2}{*}{ Dates surveyed } & \multicolumn{3}{|c|}{ Plasmodium vivax } & \multicolumn{3}{|c|}{ Plasmodium falciparum } & \multicolumn{3}{|c|}{ Plasmodium spp. } \\
\hline & Number & Risk ratio (95 \% Cl] & $P$-value & Number & Risk ratio $(95 \% \mathrm{Cl}$ & $P$-value & Number & Risk ratio $(95 \% \mathrm{Cl}$ & $P$-value \\
\hline $\begin{array}{l}\text { Jan-Mar, } 2008 \text { (Baseline, } \\
n=5585 \text { ) }\end{array}$ & 460 & Reference & - & 299 & Reference & - & 761 & Reference & - \\
\hline Jan-Mar, $2009(n=3600)$ & 172 & $0.58(0.49-0.69)$ & $<0.0001$ & 184 & $0.95(0.80-1.14)$ & 0.6452 & 358 & $0.73(0.65-0.82)$ & $<0.0001$ \\
\hline Jan-Mar, $2010(n=5090)$ & 255 & $0.61(0.52-0.71)$ & $<0.0001$ & 161 & $0.59(0.49-0.71)$ & $<0.0001$ & 429 & $0.62(0.55-0.69)$ & $<0.0001$ \\
\hline Jan-Mar, $2011(n=4069)$ & 69 & $0.21(0.16-0.26)$ & $<0.0001$ & 44 & $0.20(0.15-0.28)$ & $<0.0001$ & 113 & $0.20(0.17-0.25)$ & $<0.0001$ \\
\hline Sep-Nov, $2012(n=4561)$ & 71 & $0.19(0.15-0.24)$ & $<0.0001$ & 27 & $0.11(0.07-0.16)$ & $<0.0001$ & 98 & $0.16(0.13-0.19)$ & $<0.0001$ \\
\hline Sep-Nov, $2013(n=4517)$ & 52 & $0.14(0.11-0.19)$ & $<0.0001$ & 18 & $0.07(0.05-0.12)$ & $<0.0001$ & 70 & $0.11(0.09-0.14)$ & $<0.0001$ \\
\hline
\end{tabular}




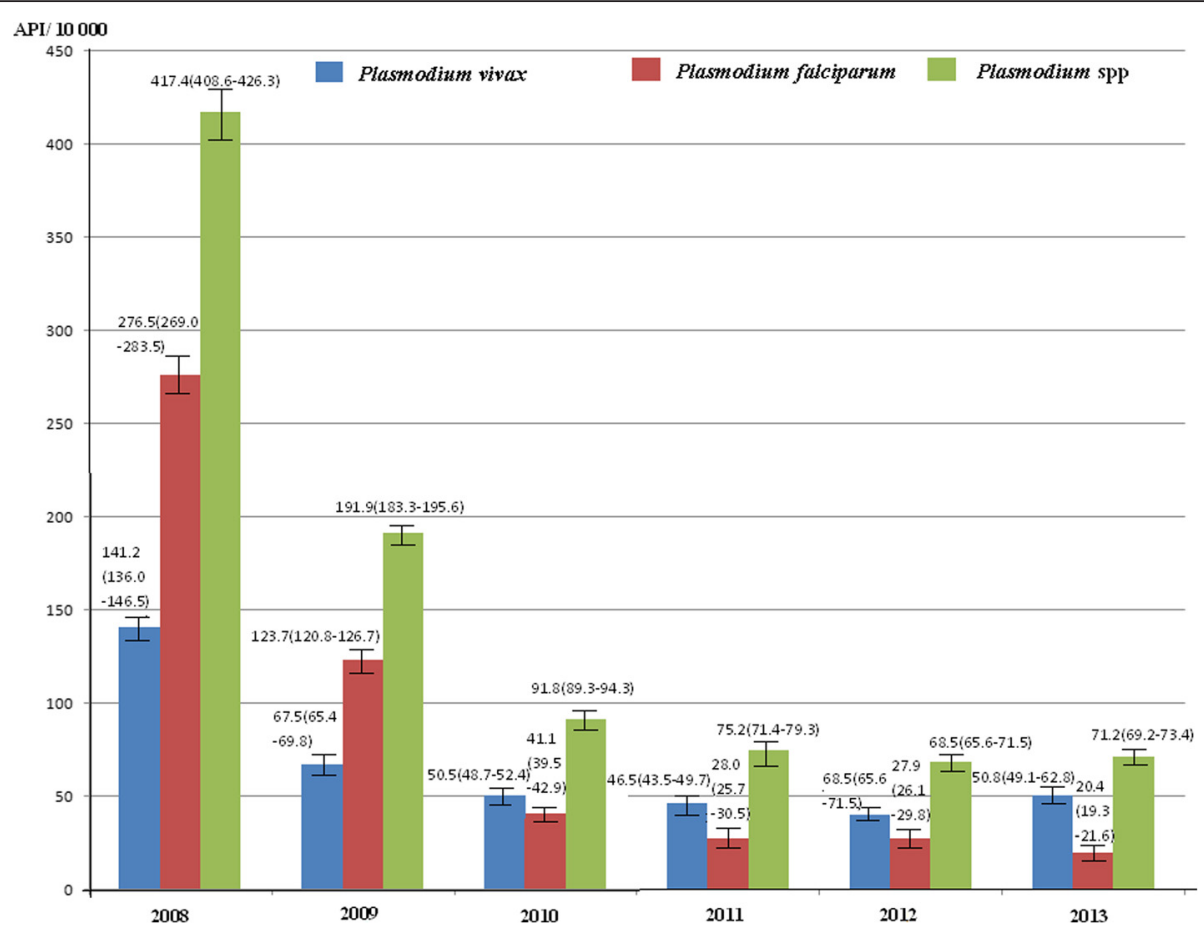

Fig. 3 Annual parasite incidences (API) in the five special regions of Myanmar from 2008 to 2013, based on Health Information System of HPA

\section{Eliminating malaria in china}

Most people of 19 border counties in China were at a high risk of malaria infection prior to the interventions. The API was 19.6 (95\% CI, 19.2-20.0) per 10000 person-years in 2006, and then the API remarkably declined to 0.9 (95\% CI, 0.8-1.0) per 10000 person-years in 2013 (Fig. 4). From 2006 to 2013, the number of imported malaria cases of Yunnan Province of China from Myanmar was 4 340, 2 291, 1 566, 1 524, 1 258, 734,388 , and 356, respectively. The proportion of imported cases in total malaria cases increased from 48.9 \% (95 \% CI, 47.9-50.0 \%) in 2006 to $84.2 \%$ (95\% CI, 80.3-87.5 \%) in 2013 (Fig. 4). Compared to the baseline (API in 2006), the IRR was only 0.05 (95\% CI, 0.04-0.05) in 2013 (Table 4), which equates to a reduction of the malaria burden by $95.0 \%$. These indicate major progress towards elimination.

The director of the Health Bureau of Mang Shi City, the former headmaster of Zhe Fang Middle School said that "The project had made impressive progress in reducing the malaria burden. Malaria transmission among school children has been a major problem prior to the intervention; but malaria was scarcely following the implementation of the GFATM programs".

Malaria was accounted for 40-50\% of total communicable diseases prior to the intervention. Stakeholders in both Mengla County and Jinghong City listed malaria as the most important public health problem before the GFATM program. Notably, they had difficulty to rank malaria at all after the intervention. Stakeholders in Mengla

Table 3 Change of annual parasite incidence rate ratios based on project reports in five special regions of Myanmar from 2008 to 2013

\begin{tabular}{|c|c|c|c|c|c|c|c|c|c|}
\hline \multirow[t]{2}{*}{ Years $\left(n=\right.$ person-years $\left.{ }^{a}\right]$} & \multicolumn{3}{|c|}{ Vivax malaria } & \multicolumn{3}{|c|}{ Falciparum malaria } & \multicolumn{3}{|c|}{ All confirmed malaria } \\
\hline & $\begin{array}{l}\text { Number of } \\
\text { cases }\end{array}$ & $\begin{array}{l}\text { Rate ratio } \\
(95 \% \text { CI }\end{array}$ & $P$-value & $\begin{array}{l}\text { Number of } \\
\text { cases }\end{array}$ & $\begin{array}{l}\text { Rate ratio } \\
\text { (95\% CI }\end{array}$ & $P$-value & $\begin{array}{l}\text { Number of } \\
\text { cases }\end{array}$ & $\begin{array}{l}\text { Rate ratio } \\
(95 \% \text { C] }\end{array}$ & $P$-value \\
\hline 2008 (Baseline, $n=195$ 849) & 2765 & Reference & - & 5409 & Reference & - & 8174 & Reference & - \\
\hline $2009(n=544876)$ & 3680 & $0.48(0.46-0.50)$ & $<0.0001$ & 6738 & $0.45(0.43-0.46)$ & $<0.0001$ & 10455 & $0.46(0.45-0.47)$ & $<0.0001$ \\
\hline $2010(n=554143)$ & 2798 & $0.36(0.34-0.38)$ & $<0.0001$ & 2279 & $0.15(0.14-0.16)$ & $<0.0001$ & 5084 & $0.22(0.21-0.23)$ & $<0.0001$ \\
\hline $2011(n=187803)$ & 873 & $0.33(0.31-0.36)$ & $<0.0001$ & 526 & $0.10(0.09-0.11)$ & $<0.0001$ & 1413 & $0.18(0.17-0.19)$ & $<0.0001$ \\
\hline $2012(n=308067)$ & 1251 & $0.29(0.27-0.31)$ & $<0.0001$ & 860 & $0.10(0.09-0.11)$ & $<0.0001$ & 2111 & $0.16(0.16-0.17)$ & $<0.0001$ \\
\hline $2013(n=626125)$ & 3181 & $0.36(0.34-0.38)$ & $<0.0001$ & 1279 & $0.07(0.07-0.08)$ & $<0.0001$ & 4460 & $0.17(0.16-0.18)$ & $<0.0001$ \\
\hline
\end{tabular}

${ }^{\mathrm{a}}$ The GFATM stopped its Chinese operations between May and December, 2011, HPA's Health Information System in the five special regions of Myanmar established and maintained by the GFATM grants, could run only for four months (January to April), $n=4$ X 563409/12=187803 person-years. In 2012, HPA spent the first half of the year to recruit new staff and restore project activities, $n=6 \times 572678 / 12=286339$ person-years 


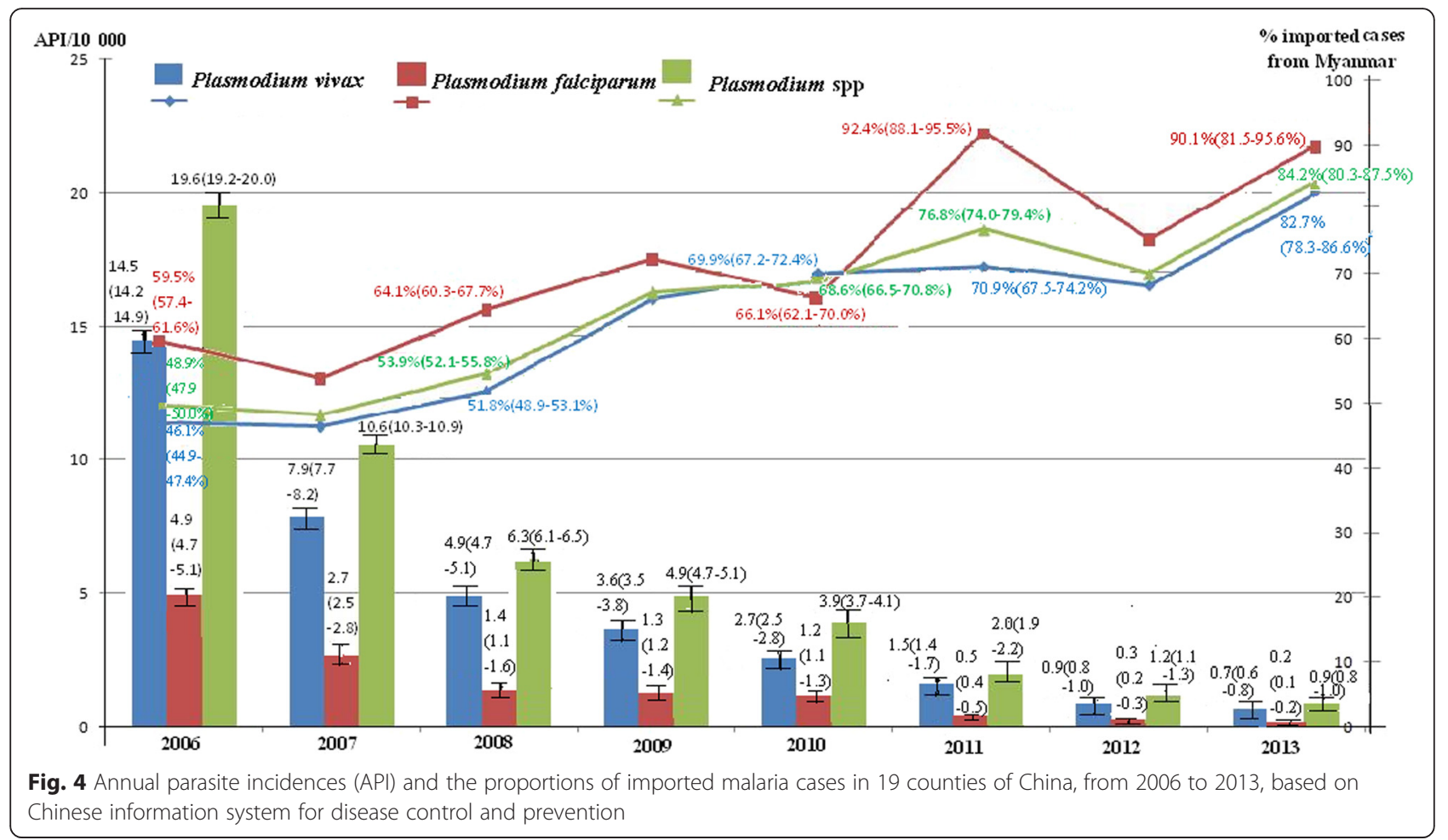

County said that "Malaria was no longer a public health problem because there was only one or two locally infected malaria cases each year in this county with a population of 310 000". However, stakeholders in Jinghong City said that "Malaria was still a major public health problem despite the fact that there were only from three to five imported malaria cases each year. Existing malaria vectors, cross-border migration of population and imported malaria from neighboring countries may reestablish malaria transmission again".

As results of surveillance of drug sensitivity, six hundred and three patients with Plasmodium vivax infections treated with chloroquine were completed a 28 day follow- up. The fever clearance time (FCT) and asexual parasite clearance times (APCT) were, respectively, $22.2 \pm 10.2$ and $38.1 \pm 12.6 \mathrm{~h}$. A total of 594 (98.5\%, $95 \%$ CI, 97.2-99.3\%) were adequate clinical and parasitological response (ACPR), and only nine (1.5\%, $95 \%$ CI $0.7-2.8 \%)$ patients were late clinical failure (LCF). The proportions of ACPR did not change significantly from 2008 to $2013\left(X^{2}=0.86\right.$, $P=0.6496$ ) [13]. Two hundred and forty three $P$. falciparum malaria patients treated with DP were completed a 42 day follow-up. The FCT and APCT were, respectively, $36.5 \pm 10.9$ and $43.5 \pm 11.8 \mathrm{~h}$, and there was an increasing trend of both FCT $(F=268.41, P<0.0001)$ and APCT $(F=$ 88.6, $P<0.0001)$ from 2007 to 2013 . Eight (3.3\%, $95 \% C I$,

Table 4 Change of annual parasite incidence rate ratios based on Chinese information system for disease control and prevention across 19 counties of Yunnan Province, China, from 2006 to 2013

\begin{tabular}{|c|c|c|c|c|c|c|c|c|c|}
\hline \multirow[t]{2}{*}{ Years ( $n=$ person-years] } & \multicolumn{3}{|l|}{ Vivax malaria } & \multicolumn{3}{|c|}{ Falciparum malaria } & \multicolumn{3}{|c|}{ All confirmed malaria } \\
\hline & $\begin{array}{l}\text { Number of } \\
\text { cases }\end{array}$ & $\begin{array}{l}\text { Rate ratio } \\
(95 \% \text { Cl] }\end{array}$ & $P$-value & $\begin{array}{l}\text { Number of } \\
\text { cases }\end{array}$ & $\begin{array}{l}\text { Rate ratio } \\
(95 \% \text { Cl] }\end{array}$ & $P$-value & $\begin{array}{l}\text { Number of } \\
\text { cases }\end{array}$ & $\begin{array}{l}\text { Rate ratio } \\
(95 \% \text { Cl] }\end{array}$ & $P$ - value \\
\hline 2006(Baseline, $n=4521$ 402) & 6570 & Reference & - & 2197 & Reference & - & 8874 & Reference & - \\
\hline $2007(n=4562688)$ & 3625 & $0.55(0.53-0.57)$ & $<0.0001$ & 1209 & $0.55(0.51-0.58)$ & $<0.0001$ & 4847 & $0.54(0.52-0.56)$ & $<0.0001$ \\
\hline $2008(n=4603659)$ & 2235 & $0.33(0.32-0.35)$ & $<0.0001$ & 665 & $0.30(0.27-0.32)$ & $<0.0001$ & 2903 & $0.32(0.31-0.34)$ & $<0.0001$ \\
\hline $2009(n=4644719)$ & 1686 & $0.25(0.24-0.26)$ & $<0.0001$ & 595 & $0.26(0.24-0.29)$ & $<0.0001$ & 2283 & $0.25(0.24-0.26)$ & $<0.0001$ \\
\hline $2010(n=4687896)$ & 1261 & $0.19(0.17-0.20)$ & $<0.0001$ & 570 & $0.25(0.23-0.27)$ & $<0.0001$ & 1833 & $0.20(0.19-0.21)$ & $<0.0001$ \\
\hline $2011(n=4729$ 147) & 732 & $0.11(0.10-0.11)$ & $<0.0001$ & 223 & $0.10(0.08-0.11)$ & $<0.0001$ & 956 & $0.10(0.10-0.11)$ & $<0.0001$ \\
\hline $2012(n=4770122)$ & 417 & $0.06(0.05-0.07)$ & $<0.0001$ & 137 & $0.06(0.05-0.07)$ & $<0.0001$ & 556 & $0.06(0.05-0.06)$ & $<0.0001$ \\
\hline $2013(n=4800944)$ & 342 & $0.05(0.04-0.05)$ & $<0.0001$ & 81 & $0.03(0.03-0.04)$ & $<0.0001$ & 423 & $0.05(0.04-0.05)$ & $<0.0001$ \\
\hline
\end{tabular}


1.4-6.4 \%) patients present parasitaemias on day three after medication; however they were spontaneous cure at day four without further treatment. In total, two hundred and forty one of 243 (99.2\%; $95 \%$ CI, 97.1 - $99.9 \%$ ) patients were ACPR. The proportions of ACPR did not change substantially from 2007 to $2013\left(X^{2}=2.81, P=0.7288\right)$ [14].

\section{Impact to health service system}

In China, the services of malaria diagnosis, treatment, and prevention for those who frequently travel across the China-Myanmar border were strengthened. In the five special regions of Myanmar, GFATM ensured that all residents had access to free quality-assured primary health care within two hours walking distance from their homes.

GFATM-supported training enhanced capacity of HPA staff, locally governmental health officers in Myanmar, and CDC staff in China on malaria control and project management. Four hundred and fifty-six received training in diagnoses including microscopy and RDT, and administering proper treatment. One thousand and forty communitybased workers including VMWs, MMWs and private practitioners received training in using RDT for diagnoses and dosing for malaria treatment. One thousand two hundred and ninety-eight community peer educators were trained to provide health education. Traditional birth attendants (TBA) were also trained to distribute LLINs and give consultation on malaria for pregnant women.

The interventions made a positive impact on basic health services. In China, an employee of Dan Zhu Border Malaria Post said that "the program had improved the capacity of the village clinic". In SR4 of Myanmar, both the HPA manager and the director of the health bureau said that "The local government had recruited and trained health care workers prior to the GFATM projects. However, in a case without GFATM grants, loss of health workers would collapse the local health service system".

\section{Reduced social inequality}

Communities including migrants and ethnical minorities along the China-Myanmar border are one of the vulnerable groups in the region. As a fact, populations who are living in malaria endemic areas are largely poor and marginalized, and at a higher risk of malaria infection. These core interventions improved accessibility and quality of malaria control services for them. Healthcare was offered regardless of gender. The VMWs and MMWs ensured communities in remote and highly endemic areas access to the free diagnosis and treatment. The poorest and the most vulnerable populations were given priority to receive LLINs with an additional one given to a pregnant woman through TBAs. Women were encouraged to actively participate in community health education and social mobilization, development of materials of information, education and communication
(IEC) and strategies of BCC. The results of a household survey in KSR2 conducted between June and September of 2013, indicated that the bed net to person ratio reached 1:1.96 (i.e., more than one net for every two people), and the LLIN to person ratio reached 1:2.52. People in the poorest families were much more likely to sleep under ITNs/LLINs than those in richer families [23]. These showed the positive impact of these interventions in reduction of social inequality.

\section{Discussion}

The China-Myanmar border region was once among the highest burden regions of malaria in the world. In 2003, a malaria epidemic took place in Kokang Region, Myanmar. One thousand three hundred and ninety-two new cases of malaria and 125 deaths were detected across 30 villages in three districts during 13 days,from the $7^{\text {th }}$ to $19^{\text {th }}$, November [24]. In December 2007, an active case detection was performed in Wa State, 453 febrile patients were found and tested by microscopy. The slide positivity rate was $60 \%(270 / 453)$ and $90.74 \%(245 / 270)$ of parasites were $P$. falciparum [25]. In October 2009, the parasite prevalence rate of $23.3 \%(36 / 159)$ was detected in school children and the P. falciparum prevalence rate of was $14.5 \%$ (23/ $159)$ in KR1 [26].

The five special regions of Myanmar are one of the major imported malaria sources in China. This situation seriously impedes malaria elimination in China [24-27]. The reduced parasite prevalence rate and API showed that the core interventions of the GFATM programs decreased malaria burden by $89 \%$ between 2008 and 2013 in Myanmar (Fig. 2, Tables 2 and 3); and by $95 \%$ between 2006 and 2013 in China (Fig. 3, Table 4). The number of imported malaria cases had decreased by $92 \%$, from 4340 in 2006 to 356 in 2013 in Yunnan Province of China. These efforts have promoted malaria elimination in China.

P. falciparum resistance to artemisinin has been confirmed in the GMS, all countries except China [1], and threatens control and elimination of malaria worldwide [10, 28]. Artemisinin resistance is rampant in Myanmar. The country is considered the main route through which drugresistant $P$. falciparum will spread from Southeast Asia to the Indian Subcontinent and then on to Africa [29, 30]. In the China-Myanmar border areas, artemisinin was highly efficacious for the treatment of falciparum malaria by 2013 $[14,31,32]$ and molecular markers of artemisinin resistance was not found by 2012 [33, 34]. These results indicate that these core interventions have prevented the development and spread of artemisinin resistance.

Huang et al. reported kelch13 propeller domain mutations in P. falciparum that was sampled from ChinaMyanmar border during 2009-2012 [35], however, the K13-propeller mutation was not detected in P. falciparum that was sampled from 2013 (unpublished data) 
to2014 [36]. The relative prevalence of $P$. falciparum parasites carrying K13-propeller mutations was investigated in 55 sites of ten administrative regions in Myanmar. K13-propeller mutation was discovered in most parts of Myanmar except China-Myanmar border [29]. Population size and genetic diversity of malaria parasites, and multi-clonal infections are correlated to the spread of anti-malarial resistance as well as transmission intensity [37]. Increasing access to quality-assured diagnosis and treatment with ACTs can limit drug resistance, and can contribute to outcome improvement in patients [38]. Most of these populations are 'hill tribes' on the China-Myanmar border. Poor access to quality-assured diagnosis and treatment gave rise to self-treatment and delayed standard treatment $[22,39]$. The results of a crosssectional study showed that only $32.0 \%$ of febrile patients sought treatment within $24 \mathrm{~h}$, while $79.6 \%$ of febrile patients purchased anti-malarial drugs themselves from the retail sector before the core interventions of the GFATM programs [22]. Free quality-assured diagnosis and treatment with ACTs to P. falciparum and the introduction of regulations successfully beat the chaotic anti-malarial market in the five special regions of Myanmar. BCC activities improved knowledge and awareness about seeking qualified treatment, and compliance with the standard treatment regimen. These core interventions successfully prevented drug resistance.

The China-Myanmar border is extremely porous. Chinese official border crossing stations recorded 10999677 person-times of border crossings in 2009. Political isolation has resulted in a lack of governmental health service structure in the five special regions of Myanmar [17]. BMPs in China and MDTS integrated with private and community-based facilities in Myanmar dramatically improved malaria diagnosis and treatment service.

Myanmar staffs received training in China through the project. This dramatically improved local and migrant access to quality-assured health service. BMPs and MDTS only provided malaria diagnoses and treatment before July, 2009. Patients who did not have malaria were not in position to receive any treatment of BMPs and MDTS. This led to fewer and fewer febrile patient visiting BMPs and MDTS. To ensure these facilities working effectively, BMPs and MDTS were expanded into public health facilities. The improvement in primary healthcare attracted febrile patients to visit these facilities.

Malaria Control along the international border can easily be affected by the political climate. In this project, an international NGO, HPA, implemented core interventions in the five Special Regions of Myanmar with financial support from GFATM and technical assistance from China under an agreement between China and Myanmar. This simplified the process and ensured carrying out these prompt and efficacious core interventions by avoiding complexity required for diplomatic coordination.

The study presents several limitations too. First, the evaluation was unable to address potential confounding, e. g. socioeconomic development, climate change and deforestation. However, China-Myanmar border was ever a high malaria transmission area $[1,2]$. These intensive control efforts played a dominant role in the change of the malaria situation. Second, Global Fund R6 and R10 were control projects. The requirement of covering as many areas as possible did not permit to use control groups. This leads to difficult to clearly link the declined API to each special intervention. Third, there were no appropriate governmental health service structures prior to the GFATM projects in the five Special Regions of Myanmar. HPA could only carry out some of these core interventions in parts of target areas because of a lack of trained staff and logistics in the first two project years. Some data such as the number of cases treat with CQ-PQ were unavailable in 2007, and only 193 cases of $P$. vivax malaria were treated in 2008. Fourth, the epidemiological data were sparse in five Special Regions of Myanmar prior to the intervention. Available data were mainly collected by the HPA's Health Information System. This leads to the number of malaria cases detected was fewer in 2008 than in 2009 because of the fact that the project did not completely cover all project areas in Myanmar in 2008. The system could only run for four months (January-April) because the GFATM abruptly stopped its China operations for eight months in 2011. HPA spent the first half of the year to recruit new staff and restored project activities, the project could only operate for six months (July to December) in 2012 (Table 3). API was also calculated as an indicator for evaluation in spite of this situation. A cross-sectional survey of parasite prevalence was undertaken to compensate for the limitation every year. Fifth, the cross-sectional survey of parasite prevalence was conducted from January to March when malaria transmission was lower during 2008-2011, and from September to November when malaria transmission was higher during 2012-2013 (Table 2). However, if anything, this time limitation of the cross-sectional surveys may just underestimate the impact of core interventions on malaria burden.

\section{Conclusions}

In conclusion, integrated approaches reduced about $90 \%$ of malaria burden on the China-Myanmar border. The intervention contributed to malaria elimination in China and containment of artemisinin resistance. Meanwhile, it strengthened the healthcare system and reduced social inequity of marginalized populations. However, further regional efforts and funding for cross-border interventions are still needed to achieve malaria elimination along the international border. 


\section{Additional files}

Additional file 1: Multilingual abstracts in the six official working languages of the United Nations. (PDF $757 \mathrm{~kb}$ )

Additional file 2: Figure S1. Map of project location relative to neighboring countries. Five special regions in Myanmar = Kachin Special Region I (KSR1), Kachin Special Region II (KSR2), Kokang, Shan Special Region II (Wa) and Shan Special Region IV (SR4). 12 counties of Round 6 in China (black dots) = Tengchong(TC), longchuan( $(\mathrm{CC})$, Yingjiang(YJ), Lianghe (LH), Longling(LL), Zhenkang(ZK), Gengma(GM),Cangyuan(CY), Ximeng(XM), Menglian(ML), Lancang(LC) and Menghai(MH). Seven counties of Round 10 in China (red dots)= Gongshan(GS), Fugong(FG), Lushui(LS), Ruili(RL), Luxi, Jinghong(JH) and Mengla(MLa). Table S1. Parasite prevalence in five special regions of Myanmar from 2008 to 2013. Table S2. Annual parasite incidence (API/10,000) based on project reports in five special regions of Myanmar from 2008 to 2013. Table S3. Annual parasite incidence (API/10,000) and number of imported malaria cases based on China information system for disease control and prevention (CISDCP) in 19 counties, China from 2006 to 2013. (DOC $472 \mathrm{~kb}$ )

\section{Abbreviations}

ACPR, Adequate clinical and parasitological response; ACT, Artemisinin- based combination therapy; APCT, Asexual parasite clearance times; API, Annual parasite incidence; BCC, Behavior change communication; BMP, Border malaria posts; CDC, Center for disease control and prevention; Cl, Confidence interval; CISDCP: Chinese Information System for Disease Control and Prevention; CQ$P Q$, Chloroquine- primaquine; DP, Dihydroartemisinin-piperaquine; FCT, Fever clearance time; FGD, Focus group discussion; GFATM, The Global Fund to fight AIDS, Tuberculosis and Malaria; GMS, Greater Mekong Subregion; HPA, Health Poverty Action; IEC, Information, education and communication; IRR, Annual parasite incidence rate ratio; ITN, insecticide-treated net; K13, Kelch gene on chromosome 13; KR2, Kachin Special Region II,Myanmar; LCF, Late clinical failure; LLIN, long lasting insecticidal net; MDTS, Malaria diagnoses and treatment station; MMW, Migrant malaria worker; NGO, Non-government organization; PPR, Parasite prevalence rate; RDT, Rapid diagnosis test; RR, Risk ratio; SDI, Semistructured in-depth interviews; SR4, Shan Special Region IV,Myanmar; TBA, Traditional birth attendant; VMW, Village malaria worker.

\section{Acknowledgements}

We thank all those participants for their contribution of time and patience in the study. The opinions expressed are those of the authors and do not necessarily reflect those of the author affiliated and funding agencies. We commemorate Dr Bo Li and give special acknowledgement to his relatives for his contribution to the health of marginalized populations. Dr Li joined the project since 2008. He is one of proposal writers for the tenth round of GFATM grant to China, and the HPA project manager of Kokang Region, Myanmar. He was deceased in a traffic accident in the work field on January 30, 2015.

\section{Funding}

This data quantitative collection was supported by the sixth (CHN-607-G09$\mathrm{M})$ and the tenth (CHN-011-G15-M) grants of the Global Fund to fight AIDS, Tuberculosis and Malaria (GFATM) to China. The qualitative study was supported by the tenth (CHN-011-G15-M) grants of the GFATM to China. Writing the manuscript was supported by the National Natural Science Foundation of China (NSFC/81560543).

\section{Availability of data and materials}

Software Epi Info 7: http://www.cdc.gov/epiinfo/.

Software TAMS 3.0: http://tamsys.sourceforge.net/.

Additional file 2

\section{Authors' contributions}

JWX was the principal writer of the proposals of the sixth and tenth rounds of GFATM grants, and he designed the protocol of investigation and evaluation. YL coordinated this evaluation and the implementation of the tenth round GFATM grants. HLY coordinated the implementation of the sixth and tenth round GFATM grants in 19 counties of China. ZJ coordinated the application of the sixth and tenth round GFATM grants and the implementation in Myanmar. ZXZ coordinated the application and the implementation of the sixth GFATM grant in China. YMY coordinated the evaluation and the implementation of the tenth round GFATM grants in 7 counties of China. HNZ coordinated the implementation of the sixth round GFATM grant in 12 counties of China. HXL, BFL and PYW collected the data from 19 counties of China. HL monitored the drug resistance. XJW, HZ and $H X L$ conducted the qualitative study. XYX and JQD respectively organized the implementation of the sixth and tenth rounds of GFATM grants in five special regions of Myanmar. YZ, XYS, BL, JYL and YHT coordinated the crosssectional survey for parasite prevalences each year. JWX and JH wrote the first draft of the paper. All authors read and approved the final manuscript.

\section{Competing interests}

The authors declare that they have no competing interests.

\section{Ethics approval and consent to participate}

The Ethics Committee of Yunnan Institute of Parasitic Diseases (YIPD), China convened a meeting to review project "Research of risk factor for malaria infection along cross China-Myanmar border" on 12 March 2015. As a part of this project, "Malaria control along cross China-Myanmar Border during 2007-2013: an integrated impact evaluation" is to evaluate the impact of malaria control intervention China-Myanmar Border from 2007 to 2013. It does not involve the use of any animal or human samples, however it analyses and reports human data. The ethics committee of YIPD approved the study [Reference number: YIPD-EC-2015 (02)] and required to explain and disclose the purpose and procedures of the qualitative study to all participants, to ensure obtain verbal consent from participants. The participants had the right to withdraw from the study at any time. All results must be kept confidential and unlinked to any identifying information.

\section{Author details}

${ }^{1}$ Yunnan Institute of Parasitic Diseases, Yunnan Provincial Center of Malaria Research, Yunnan Provincial Collaborative Innovation Center for Public Health and Disease Prevention and Control, Yunnan Provincial Key Laboratory of Vector-borne Diseases Control and Research, Puer 665000, China. ${ }^{2}$ Yunnan Provincial Health and Family Planning Commission, Kunming 650200, People's Republic of China. ${ }^{3}$ Yunnan Office of Health Poverty Action, Kunming 650041, People's Republic of China. ${ }^{4}$ World Health Organization Regional Office for the Western Pacific, P.O. Box 29321000 Manila, Philippines. ${ }^{5}$ Foundation for Innovative New Diagnostics, 1216 Cointrin, Geneva, Switzerland.

Received: 29 November 2015 Accepted: 11 July 2016

Published online: 10 August 2016

\section{References}

1. World Malaria Report 2014. Geneva: World Health Organization; 2014

2. Cui L, Yan G, Sattabongkot J, Cao Y, Chen B, Chen X, Fan Q, Fang Q, Jongwutiwes S, Parker D, Sirichaisinthop J, Kyaw MP, Su XZ, Yang H, Yang Z, Wang B, Xu J, Zheng B, Zhong D, Zhou G. Malaria in the Greater Mekong Subregion: heterogeneity and complexity. Acta Trop. 2012;121(3):227-39.

3. Delacollette C, Souza C, Christophel E, Thimasarn K, Abdur R, Bell D, Dai TC, Gopinath D, Lu S, Mendoza R, Ortega L, Rastogi R, Tantinimitkul C, Ehrenberg J. Malaria trends and challenges in the Greater Mekong Subregion. Southeast Asian J Trop Med Public Health. 2009;40(4):674-91.

4. Ren M, Scano F, Sozi C, Schwartlander B. The Global Fund in China: success beyond the numbers. Lancet Glob Health. 2015;3:75-7.

5. Ministry of Health, People's Republic of China. Malaria Elimination Action Plan 2010-2020. Beijing: Ministry of Health of China; 2010. Accessed 27 Jun 2010. (in Chinese).

6. WHO. Malaria in the Greater Mekong Subregion: regional and country profiles. Bangkok: The Representative Office for World Health Organization to Thailand; 2008. Accessed 26 Oct 2014.

7. Beyrer C, Lee TJ. Responding to infectious diseases in Burma and her border regions. Confl Health. 2008;2:2.

8. Emergency Response to Artemisinin Resistance in the Greater Mekong Subregion: RegionalFramework for Action 2013-2015. Geneva: World Health Organization; 2014.

9. Greenwood B. Treatment of malaria - a continuing challenge. N Engl J Med. 2014;371:474-5.

10. Dondorp A, Nosten F, Yi P. Artemisinin resistance in Plasmodium falciparum malaria. N Engl J Med. 2009;361:455-67. 
11. Phyo AP, Nkhoma S, Stepniewska K. Emergence of artemisinin-resistant malaria on the western border of Thailand: a longitudinal study. Lancet. 2012;379:1960-6.

12. Ashley EA, Dhorda M, Fairhurst RM. Spread of artemisinin resistance in Plasmodium falciparum malaria. N Engl J Med. 2014;371:411-23.

13. Liu $H$, Yang $H L$, Tang LH, Li XL, Huang F, Wang JZ, Li CF, Wang HY, Nie RH, Guo XR, Lin YX, Li M, Xu JW. Monitoring Plasmodium vivax chloroquine sensitivity along China-Myanmar border of Yunnan Province, China during 2008-2013. Malar J. 2014;13:364.

14. Liu H, Yang HL, Tang LH, Li XL, Huang F, Wang JZ, Li CF, Wang HY, Nie RH, Guo XR, Lin YX, Li M, Wang J, XU JW. In vivo monitoring of dihydroartemisinin-piperaquine sensitivity in Plasmodium falciparum along the China-Myanmar border of Yunnan Province, China during 2007-2013. Malar J. 2015;14:47

15. Ministry of Health, People's Republic of China. The implementation plan of the tenth China's malaria program for the Global Fund to fight AIDS, Tuberculosis and Malaria. Beijing: Ministry of Health of China; 2012. Accessed 20 February 2012. (in Chinese).

16. Yang W, Li Z, Lan Y. A nationwide web-based automated system for outbreak early detection and rapid response in China. Manila: WPSAR, World Health Organization Regional Office for the Western Pacific; 2011.

17. Wang RB, Zhang J, Zhang QF. Malaria baseline survey in four special regions of northern Myanmar near China: a cross-sectional study. Malar J. 2014;13:302.

18. WHO. Assessment and Monitoring of antimalarial drug efficacy for The Treatment of Uncomplicated Falciparum Malaria. Geneva: World Health Organization; 2003 (WHO/RBM/HTM/2003.50).Accessed 20 Dec 2014

19. WHO. Method for surveillance of antimalarial drug efficacy. Geneva: World Health Organization; 2009. Accessed 20 Dec 2014.

20. WHO. Methods and Techniques for Clinical Trials on Antimalarial Efficacy. Genotyping to Identify Parasite Populations. Geneva: World Health Organization; 2008. Accessed 20 Dec 2014

21. Xu JW, Liao YM, Liu H, Nie RH, Havumaki J. Use of bed nets and factors that influence bed net use among Jinuo ethnic minority in Southern China. PLoS One. 2014;9:e103780. doi:10.1371/journal.pone.0103780.

22. Xu JW, Xu QZ, Liu H, Zeng YR. Malaria treatment-seeking behaviour and related factors of Wa ethnic minority in Myanmar: a cross-sectional study. Malar J. 2012;11:417

23. Liu H, Xu JW, Guo XR, Havumaki J, Yu GC, Zhou DL. Coverage, use and maintenance of bed nets and related influence factors in Kachin Special Region II, Northeastern Myanmar. Malar J. 2015;14:212.

24. Li HX, Zhang ZX, Chen GW, Chen ZW. Investigation into an outbreak of malaria in Lincang profecture on China - Myanmar border and the neighboring area outside China. Chin Trop Med. 2005;5:55-7 (in Chinese).

25. Liu H, Nie RH, Li CF, SunYH LGS. Active detection of malaria Cases in Myanmar Wa Ethnical villages of China-Myanmar border. Parasit Infect Dis. 2009;12:6-8 (in Chinese)

26. Liu H, Li XL, Chen H, Deng C, Long K, Kong L. A cross-sectional malaria survey in Mangwen district, Kachin State, Myanmar. Chin J Pathogen Bio. 2011;6:55-9 (in Chinese).

27. Chen GW, Li HX, Lin YX. Horizontal survey on the epidemiological characteristics of malaria in Laiza city of the second special administrative region of Kachin State of Myanmar, a Chinese-Myanmar border area. Chin J Vector Bio Control. 2012;23:352-256 (in Chinese).

28. Ariey F, Witkowski B, Amaratunga C. A molecular marker of artemisininresistant Plasmodium falciparum malaria. Nature. 2014:505:50-5.

29. Tun KM, Imwong M, Lwin K. Spread of artemisinin-resistant Plasmodium falciparum inMyanmar: a cross-sectional survey of the K13 molecular Marker. Lancet Infect Dis. 2015;15:415-21.

30. Menard D, Ariey F. Towards real-time monitoring of artemisinin resistance. Lancet Infect Dis. 2015;15:367-8.

31. Sun XD, Zhang ZX, Liu DQ. Clinical study on the efficacy of dihyroartemisinin and piperaquine in treatment of multi-drug resistant Plasmodium falciparum. Chin Trop Med. 2006;6:211-2 (in Chinese).

32. Liu H, Yang HL, Zhang J, Li CF, Nie RH, Wang HY. Clinical trial on efficacy of compound dihydroartemisinin-piperaquine for treatment of uncomplicated falciparum malaria in Myanmar. Chin J Parasit Parasitic Dis. 2011;29: 296-8 (in Chinese)

33. Huang F, Tang LH, Yang HL, Zhou SS, Sun XD, Liu H. Therapeutic efficacy of artesunate in the treatment of uncomplicated Plasmodium falciparum malaria and anti-malarial, drug-resistance marker polymorphisms in populations near the China-Myanmar border. Malar J. 2012;11:278.
34. Wang Z, Parker D, Meng $H$. In vitro sensitivity of Plasmodium falciparum from China-Myanmar border area to major ACT drugs and polymorphisms in potential target genes. PLoS One. 2012;7:e30927. doi:10.1371/journal. pone.0030927.

35. Huang F, Takala-Harrison S, Jacob CG, Liu H, Sun X, Yang H, Nyunt MM, Adams M, Zhou S, Xia Z, Ringwald P, Bustos MD, Tang L, Plowe CV. A single mutation in $\mathrm{k} 13$ predominates in southern china and is associated with delayed clearance of plasmodium falciparum following artemisinin treatment. J Infect Dis. 2015;212(10):1629-35. doi:10.1093/infdis/jiv249.

36. Liu H, Xu JW, Yang HL, Li M, Sun CD, Yin YJ, et al. Investigation and control of a Plasmodium falciparum malaria outbreak in Shan Special Region II of Myanmar along the China-Myanmar Border from June to December 2014 Infect Dis Poverty. 2016;5:32.

37. Yuan LL, Zhao H, Lanou WL. Plasmodium falciparum populations from northeastern Myanmar display high levels of genetic diversity at multiple antigenic loci. Acta Trop. 2013;125:53-9.

38. WHO. Guidelines for the treatment of malaria (2nd Ed). Geneva: World Health Organization; 2011. Accessed 20 Dec 2014

39. Sonkong $K$, Chaiklieng S, Neave P, Suggaravetsiri P. Factors affecting delay in seeking treatment among malaria patients along Thailand-Myanmar border in Tak Province, Thailand. Malar J. 2015;14:3.

\section{Submit your next manuscript to BioMed Central and we will help you at every step:}

- We accept pre-submission inquiries

- Our selector tool helps you to find the most relevant journal

- We provide round the clock customer support

- Convenient online submission

- Thorough peer review

- Inclusion in PubMed and all major indexing services

- Maximum visibility for your research

Submit your manuscript at www.biomedcentral.com/submit
) Biomed Central 